\title{
Alkali tellurite glasses: Bonding, structure and conductivity*
}

\author{
C S SUNANDANA \\ School of Physics, University of Hyderabad, Hyderabad 500046, India \\ MS received 26 March 1996
}

\begin{abstract}
The nature of the $\mathrm{Te}-\mathrm{O}$ bond and charge transfer between alkali atoms and $\mathrm{Te}-\mathrm{O}$ polyhedra in alkali-tellurite glasses is discussed in connection with ion transport in these glasses. Experimental results on XPS, neutron and Raman scattering support the empirical picture developed here. The overall kinetics of the interchange transport mechanism for conduction in single and mixed alkali-tellurite glasses is apparently determined by the kinetics of the alkali ion--glass former ion interchange - an aspect to be probed experimentally.
\end{abstract}

Keywords. Alkali tellurite glasses; bonding; structure; conductivity.

\section{Introduction}

Alkali tellurite glasses $-\mathrm{A}_{2} \mathrm{O}-\mathrm{TeO}_{2}(\mathrm{~A}=\mathrm{Li}$. $\mathrm{Na}, \mathrm{K})$ have (i) relatively low melting points (but are non-hygroscopic), (ii) high refractive indices $(>2)$, (iii) large dielectric constants, (iv) good IR transmission coefficients, (v) high thermal expansion coefficients and (vi) reasonably good ionic conductivity.

These characteristics emerge from unique short-range structural effects, electronic structure and local chemical bonding peculiar to these glasses. As far as electrical conductivity is concerned, non-bridging oxygens, electronic charge transfer and ion hopping play an important role - both in 'single alkali' and 'mixed alkali' tellurite glasses.

In this paper, we discuss aspects of chemical bonding in, and local structure of, alkali tellurite glasses as they relate to aspects of ionic conductivity. We shall utilize experimental data on neutron and Raman scattering to arrive at an acceptable model for ion conduction in single alkali and mixed-alkali tellurite glasses.

\subsection{Bonding}

Local chemical bonding holds the key to global structure - whether for crystalline or non-crystalline solids. More precisely, it is the extent of charge transfer among the constituent elements that decides the geometry and stability of the glass-building block. To begin with, one could consider the nature of the Te-O bond from three points of view: (i) electronegativity difference, (ii) radius ratio and (iii) bond energy in relation to boiling point.

The Pauling electronegativities $(\chi)$ of Te and $\mathrm{O}$ are 2.21 and 3.44 respectively. Thus Te is electropositive $\left(\chi_{\mathrm{Te}}<\chi_{0}\right)$ and charge should transfer from Te to $O$. Furthermore, $\Delta \chi=1.23$ (which corresponds to an ionicity of $39 \%$ ) is less than 1.90 and makes $\mathrm{TeO}_{2}$ a glass former according to Stanworth (1971). Furthermore, this number expressed in $\mathrm{k}$ cal represents the enthalpy of formation of the Te-O bond (Pauling 1975). When we mix an alkali oxide $\mathrm{Li}_{2} \mathrm{O}$ and $\mathrm{TeO}_{2}$ and melt the mixture to form a glass, the bonds are

\footnotetext{
*Based on a talk at the Materials Science Symposium of the 83rd Indian Science Congress, Patiala, 1996.
} 
scrambled and frozen. The average electronegativity difference $\left(\Delta \chi_{\mathrm{Li}-\mathrm{o}}+\Delta \chi_{\mathrm{Te}-\mathrm{o}}\right) / 2$ $=(2.5+4.5) 2=1.95$, yet close to the glass forming criterion but more ionic than $\mathrm{TeO}_{2}$ glass. The radius ratio $r_{\mathrm{Te}(4+)} / r_{\mathrm{O}^{2-}}=84 \mathrm{pm} / 140 \mathrm{pm}=0.6$ is favourable for the formation of an octahedron of oxygens around $\mathrm{Te}$. Interestingly enough the sum of tetrahedral covalent radii of $\mathrm{Te}(132 \mathrm{pm})$ and oxygen $(66 \mathrm{pm})$ is $1.98 \AA$ which is found to be close to the average $\mathrm{Te}-\mathrm{O}$ distance in crystalline and glassy $\mathrm{TeO}_{2}$. In fact, neutron scattering experiments on $\mathrm{Li}_{2} \mathrm{O}-\mathrm{TeO}_{2}$ (Neov et al 1979) put the $\mathrm{Te}-\mathrm{O}$ distance as $1.93 \AA$. There is thus, a delicate balance of covalency and ionicity in $\mathrm{TeO}_{2}$ which makes the bonding unique in this 'stand-alone' glass former (Lambson et al 1984). Finally consider the ratio of the bond strength to melting point an important criterion for glass formation. This value for $\mathrm{TeO}_{2}$ is lowest $(0.040 \mathrm{kcal} / \mathrm{mol} \mathrm{K})$ among canonical glass formers $\left(0.053\right.$ for $\mathrm{SiO}_{2}$ and 0.130 for $\left.\mathrm{B}_{2} \mathrm{O}_{3}\right)$.

The basic octahedral geometry inferred from the above empirical considerations is found to be correct. Recent work on the electronic structure of tellurite glasses based on the distorted rutile structure of paratellurite has shown that the building block in tellurite glasses is indeed a $\mathrm{Te}-\mathrm{O}_{6}$ octahedron albeit at distorted one, the distortion arising from $5 s-5 p$ hybridization of the Te atom and leading to four strong $\mathrm{Te}-\mathrm{O}$ bonds (Suehara et al 1995). Very interesting experimental confirmation of this has come from the valence bond spectra (XPS) of $\alpha-\mathrm{TeO}_{2}$ (Suehara et al 1994) and from the ESR of a hole defect in electron-irradiated paratellurite (Watterich et al 1985). Thus the ambiguity over the structural basis of $\mathrm{TeO}_{2}$ i.e. whether the building block is derived from $\alpha-\mathrm{TeO}_{2}$ or $\beta-\mathrm{TeO}_{2}$ has been resolved in favour of the former.

\subsection{Structure}

The structure of alkali-tellurite glasses is based on (i) the Te-O building block and (ii) the alkali-oxygen bonding, or, more precisely the alkali- $\mathrm{TeO}_{n}$ bonding. The latter assumes great importance in view of the difference in the properties of alkali atoms and thus differences in alkali-oxygen bonding. As the electronic charge transfer depends on these properties (Suehara et al 1995), one has to focus on the changes in charge transfer as one alters the concentration of alkali oxide in a single alkali tellurite glass or as one replaces one alkali by another in a mixed alkali-tellurite glass. The subtle differences in alkali-oxygen charge transfer makes the glass structure inhomogeneous by creating a number of $\mathrm{Te}-\mathrm{O}_{n}(3 \leqslant n \leqslant 6)$ and thus the structural changes arise. In turn, these latter influence the ion transport paths and decide the conductivity behaviour.

The electronegativity of the alkali atoms in alkali tellurite glasses is smaller than those of Te and $\mathrm{O}\left(\mathrm{e} . \mathrm{g} . \chi_{\mathrm{Li}}=1.0, \chi_{\mathrm{Na}}=0.9\right.$ while $\chi_{\mathrm{Te}}=2.1$ and $\chi_{\mathrm{O}}=3.5$ ). Therefore the charge transfer must take place from say $\mathrm{Li}$ to $\mathrm{TeO}_{n}$ units. What is the maximum charge transfer? Assuming $\mathrm{Li}_{2} \mathrm{TeO}_{3}$ (and $\mathrm{Na}_{2} \mathrm{TeO}_{3}$ ) as the corresponding crystalline 'precursors', the maximum charge transfer would be 2 units. If the net charge on $\mathrm{TeO}_{6}$ is assumed to be $8 \delta^{-}$(taking $\mathrm{Te}^{4 \delta+}$, and $\mathrm{O}^{2 \delta-}$ as charges on individual ions, $\delta$ being partial ionic character) then a maximum charge transfer of 2 units would mean $8 \delta=\sim 6$ or $\delta=0.75$. This would make Te triply positive $\left(\mathrm{Te}^{3+}\right)$ and oxygen $\mathrm{O}^{1 \cdot 5-}$ Suppose the charge transfer is 1 , then $8 \delta=\sim 7$, or $\delta=0.9$ which would yield $\mathrm{Te}^{3.6+}$ and $\mathrm{O}^{1 \cdot 8-}$. The charge transfer is expected to vary continuously from 0 to 1 as the alkali concentration is increased from 0 to the maximum permissible value. This progressive charge transfer, however would not result in a monotonic change in local structure but 
would lead at some specific concentration of the alkali ion to the formation of non-bridging oxygens (NBO) in significant numbers. From the point of view of electrical conductivity this would signal a stage when the activation energy for ion migration would change little with concentration. The recent dynamic angle spinning ${ }^{23} \mathrm{Na} \mathrm{NMR}$ study on $\mathrm{Na}_{2} \mathrm{O}-\mathrm{TeO}_{2}$ glasses (Tagg et al 1995) has shown that there is a change in coordination of $\mathrm{Na}$ from about six at low modifier concentration (10 to $18 \mathrm{~mol} \%$ ) to about five at high concentration ( $>18 \mathrm{~mol} \%$ ), which has been interpreted as due to the onset of NBO formation. Similar studies on $\mathrm{Li}_{2} \mathrm{O}-\mathrm{TeO}_{2}$ glasses using ${ }^{7} \mathrm{Li}$ NMR probe would be highly desirable.

This net charge transfer is also affected when one alkali atom $(\mathrm{Li})$ is partially replaced by another $(\mathrm{Na})$ atom. The chemical homogeneity of the glass would be naturally affected by this localized charge transfer, by way of the occurrence of different types of $\mathrm{TeO}_{n}$ units randomly distributed over the glass network. These changes would automatically be reflected in the creation of many types of conduction paths leading to different conduction mechanisms in single alkali and mixed alkali tellurite glasses. Thus the addition of alkali (or an alkaline earth oxide) to $\mathrm{TeO}_{2}$ would lead to a 'depolymerization' of the $\mathrm{TeO}_{2}$ framework (Yamamoto 1994) and thus create alternative diffusion paths for alkali-ion migration. These changes have been monitored by Raman scattering experiments on rapidly quenched alkali-tellurite glasses (Tatsumisago et al 1994).

In an earlier paper (Sunandana 1995) we had raised three questions with regard to the ion interchange transport process as a viable ion-conduction mechanism in single alkali and mixed alkali-tellurite (and other) glasses. These questions relating to the energetics, kinetics and 'local structural collapse' dynamics have been subsequently examined in some detail (Sunandana 1996). There appears to be a hierarchy of energies

$$
E_{\mathrm{t}}>E_{\mathrm{i}}>E_{\mathrm{hf}}>E_{\mathrm{d}}>E_{\mathrm{ha}} \text {, }
$$

where $E_{\mathrm{i}}$ is the energy required to remove an alkali (say $\mathrm{Li}^{+}$) from its interstitial position in the glass network, $E_{\mathrm{d}}$ the energy required to free $\mathrm{Li}^{+}$attached to an NBO, $E_{\mathrm{t}}$ the energy required to 'yank out' glass former ion (say $\mathrm{Te}^{4+}$ ) from its trigonal prismatic site in the network, $E_{\mathrm{hf}}$ the energy of hopping of $\mathrm{Te}$ to a bound $\mathrm{Li}^{+}$and $E_{\text {ha }}$ the energy for bound $\mathrm{Li}^{+}$to hop back to $\mathrm{Te}$.

The crucial kinetics of this process would be that for the glass former ion-bound alkali ion interchange. The rate of this interchange probably decides the overall kinetics of the problem. The 'local collapse' of the network during the ion interchange also constitutes an interesting (but complicated) process and it would be interesting to probe this experimentally, say using pulsed NMR of the ${ }^{7} \mathrm{Li}$ nucleus $(I=3 / 2)$ (Unfortunately, Te, unlike ${ }^{29} \mathrm{Si}(I=1 / 2)$ and ${ }^{11} \mathrm{~B}(I=3 / 2)$ does not possess a magnetic nucleus so one cannot do Te-NMR). Specifically the general dynamical aspect to be probed is the exchange of $\mathrm{Li}$ atoms between sites or the rotation of the relevant species (with respect to the magnetic field) that affects the NMR lineshape appreciably (Stebbins 1988).

\section{Conclusion}

The nature of the $\mathrm{Te}-\mathrm{O}$ bond and charge transfer between alkali atoms and $\mathrm{Te}-\mathrm{O}$ polyhedra in alkali-tellurite glasses has been discussed in connection with the ion transport in these glasses. Experimental results on XPS, neutron and Raman scattering 
support the empirical picture developed here. The overall kinetics of the interchange transport mechanism for conduction in single and mixed alkali-tellurite glasses is apparently determined by the kinetics of the alkali ion-glass former ion interchange an aspect to be probed experimentally.

\section{Acknowledgements}

I thank Prof. K J Rao for his invitation and the University of Hyderabad, Hyderabad for financial support.

\section{References}

Lambson E F, Sanders G A, Bridge B and El-Mallawany R A 1984 J. Nanocrystalline Solids 69117 Neov S et al 1979 J. Phys. C12 2475

Pauling L 1975 The nature of the chemical bond (New Delhi: Oxford and IBH) Ch. 3

Stanworth J E 1971 J. Am. Ceram. Soc. 5461

Stebbins J F 1988 J. Nanocrystalline Solids 106359

Suehara S et al 1994 Phys. Rev. B50 7981

Suehara S et al 1995 Phys. Rev. B51 14919

Sunandana C S 1995 Bull. Mater. Sci. 1817

Sunandana C S 1996 to be published

Tagg S L, Youngman R E and Zwanziger J W 1995 J. Phys. Chem. 995111

Tatsumisago M et al 1994 Phys. Chem. Glasses 3589

Watterich A et al 1985 Phys. Rev. B32 2533

Yamamoto H et al 1994 J. Noncrvstalline Solids 17087 